CRYSTALLOGRAPHIC COMMUNICATIONS

\section{A new pseudopolymorph of perchlorinated neopentasilane: the benzene monosolvate $\mathrm{Si}\left(\mathrm{SiCl}_{3}\right)_{4} \cdot \mathrm{C}_{6} \mathrm{H}_{6}$}

\author{
Jan Tillmann, Hans-Wolfram Lerner and Michael Bolte*
}

Received 10 December 2019

Accepted 23 January 2020

Edited by W. T. A. Harrison, University of Aberdeen, Scotland

Keywords: crystal structure; co-crystal; polymorphism; solvate.

CCDC reference: 1979631

Supporting information: this article has supporting information at journals.iucr.org/e
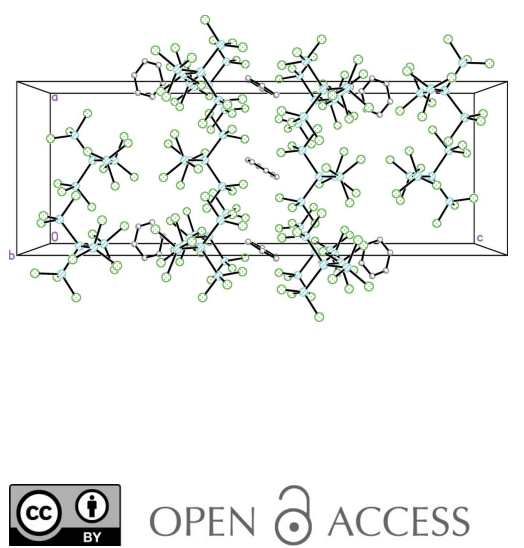

OPEN ӘACCESS
Institut für Anorganische und Analytische Chemie, Goethe-Universität Frankfurt, Max-von-Laue-Strasse 7, 60438 Frankfurt am Main, Germany. *Correspondence e-mail: bolte@chemie.uni-frankfurt.de

A new pseudopolymorph of dodecachloropentasilane, namely a benzene monosolvate, $\mathrm{Si}_{5} \mathrm{Cl}_{12} \cdot \mathrm{C}_{6} \mathrm{H}_{6}$, is described. There are two half molecules of each kind in the asymmetric unit. Both $\mathrm{Si}_{5} \mathrm{Cl}_{12}$ molecules are completed by crystallographic twofold symmetry. One of the benzene molecules is located on a twofold rotation axis with two $\mathrm{C}-\mathrm{H}$ groups located on this rotation axis. The second benzene molecule has all atoms on a general position: it is disordered over two equally occupied orientations. No directional interactions beyond normal van der Waals contacts occur in the crystal.

\section{Chemical context}

Since the 1980s, silicon hydrides, such as $\mathrm{Si}\left(\mathrm{SiH}_{3}\right)_{4}$, have attracted considerable attention as precursors for the liquid phase deposition (LPD) of silicon thin films (Nishimura et al., 1985). In this context it should be noted that the perchlorinated neopentasilane $\mathrm{Si}\left(\mathrm{SiCl}_{3}\right)_{4}\left(\mathrm{Si}_{5} \mathrm{Cl}_{12}\right)$ is easily accessible in large amounts by the amine-induced disproportionation (Meyer-Wegner et al., 2011; Tillmann et al., 2012) of perchloropolysilanes, e.g. $\mathrm{Si}_{2} \mathrm{Cl}_{6}$ or $\mathrm{Si}_{3} \mathrm{Cl}_{8}$ (Meyer-Wegner et al., 2011; Urry, 1970). Subsequent hydrogenation of $\mathrm{Si}\left(\mathrm{SiCl}_{3}\right)_{4}$ (I) then yields the neopentasilane $\mathrm{Si}\left(\mathrm{SiH}_{3}\right)_{4}$, which can be used as an LPD agent (Cannady \& Zhou, 2008) (see Fig. 1).<smiles>[SiH3][Si]([SiH3])([SiH3])[SiH3]</smiles>

In this paper we describe the structure of a new pseudopolymorph of perchlorinated neopentasilane (I), namely the benzene monosolvate $\mathrm{Si}\left(\mathrm{SiCl}_{3}\right)_{4} \cdot \mathrm{C}_{6} \mathrm{H}_{6}$, and make a comparison of its structure with those of $\mathrm{Si}\left(\mathrm{SiCl}_{3}\right)_{4}$ (Meyer-Wegner et al., 2011) and $\mathrm{Si}\left(\mathrm{SiCl}_{3}\right)_{4} \cdot \mathrm{SiCl}_{4}$ (Fleming, 1972).

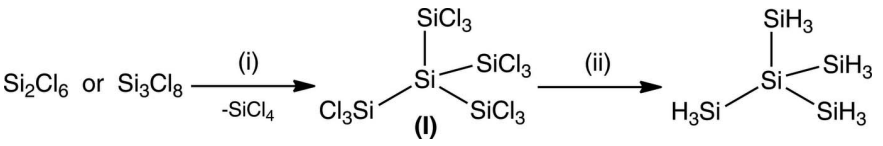

Figure 1

Amine-induced disproportionation of $\mathrm{Si}_{2} \mathrm{Cl}_{6}$ and $\mathrm{Si}_{3} \mathrm{Cl}_{8}$ : (i) $+\mathrm{NMe}_{3}$, or $\mathrm{NMe}_{2} \mathrm{Et}$, or $\mathrm{NEt}_{3}$ in benzene at room temperature; (ii) $+\mathrm{LiAlH}_{4}$ in diethyl ether at room temperature 


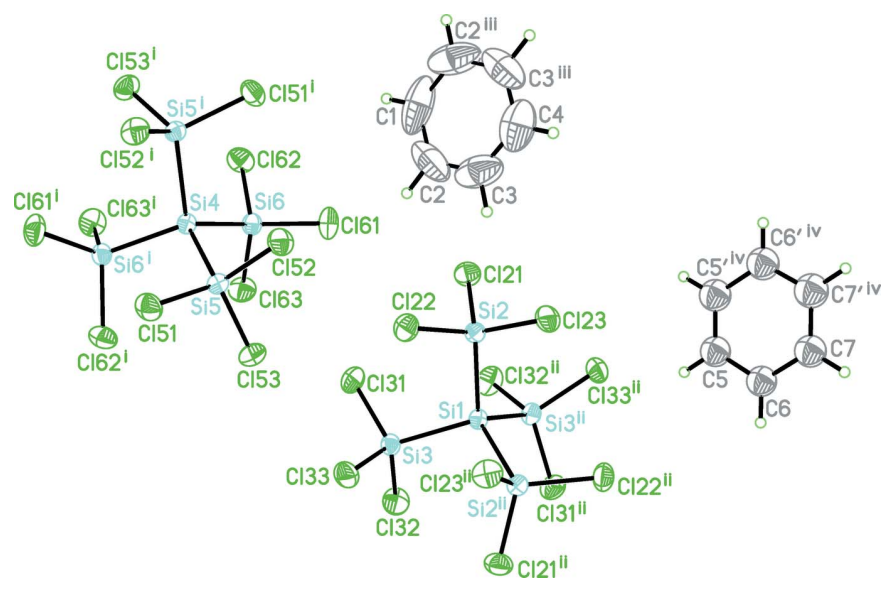

Figure 2

A perspective view of the title compound. Displacement ellipsoids are drawn at the $50 \%$ probability level. Symmetry codes: (i) $-y,-x,-z+\frac{1}{4}$; (ii) $1-y, 1-x,-z+\frac{1}{4}$; (iii) $-x, y,-z$; (iv) $1-x, y,-z$.

\section{Structural commentary}

There are two half molecules of each kind in the asymmetric unit of (I). Both $\mathrm{Si}_{5} \mathrm{Cl}_{12}$ molecules are completed by crystallographic twofold symmetry, with the rotation axes orientated in the [110] and [110] directions and the central $\mathrm{Si}$ atom located on the axis (Fig. 2). One of the benzene molecules is located on a twofold rotation axis propagating along the $a$ or $b$ axes with two $\mathrm{C}-\mathrm{H}$ groups located on this rotation axis. The second benzene molecule has all atoms on general positions: it is disordered over two equally occupied orientations about a twofold rotation axis running in the [100] and [010] directions.

\section{Supramolecular features}

A view of the molecular packing of (I) (Fig. 3) reveals that the benzene molecules fill the voids between the dodecachloropentasilane molecules. There are no identified directional intermolecular interactions.

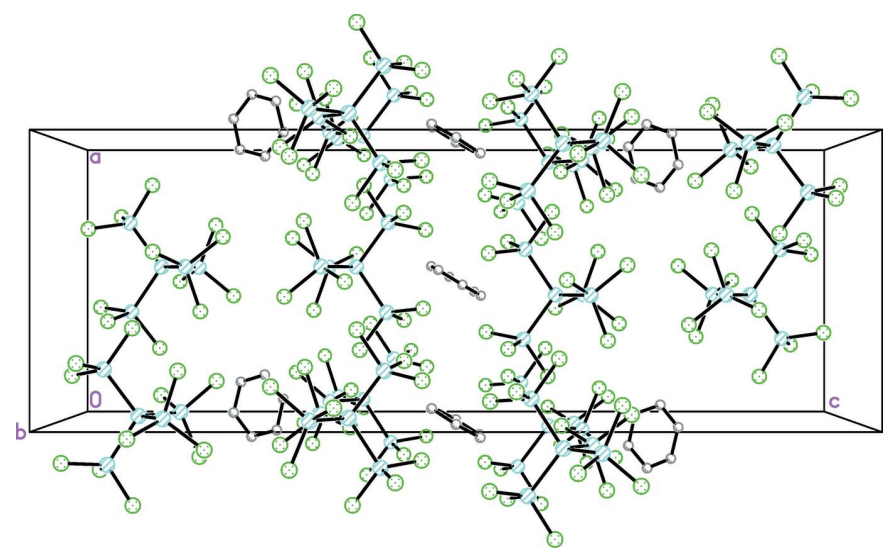

Figure 3

Packing diagram of the title compound viewed down [010].
Table 1

Bond lengths $(\AA)$ in the different structures containing $\mathrm{Si}_{5} \mathrm{Cl}_{12}$ molecules.

For (I), mean values of the two molecules are given. For (II), mean values of the non-disordered molecule are given. Because of the high symmetry of (III), there is only one value for each bond length.

\begin{tabular}{lll}
\hline & $\mathrm{Si}-\mathrm{Si}$ & $\mathrm{Si}-\mathrm{Cl}$ \\
\hline (I) & 2.324 & 2.019 \\
(II) & 2.340 & 2.026 \\
(III) & $2.332(9)$ & $1.994(7)$ \\
\hline
\end{tabular}

\section{Database survey}

There are two already known structures of dodecachloropentasilane: first, there is pure $\mathrm{Si}_{5} \mathrm{Cl}_{12}$ (Meyer-Wegner et al., 2011; CCDC deposition number 793308) and second, a cocrystal with silicon tetrachloride (Fleming, 1972; CCDC deposition number 1592571). In each of these structures, the $\mathrm{Si}_{5} \mathrm{Cl}_{12}$ molecule is located on a special position. As noted above, in (I), both molecules in the asymmetric unit are found on a twofold rotation axis. Compound (II) also crystallizes with two molecules in the asymmetric unit. One of them is located on a threefold rotation axis and the other is disordered about a special position of site symmetry $\overline{3}$. In the second molecule, it is noteworthy that only the $\mathrm{Si}$ atoms carrying the $\mathrm{Cl}$ atoms are disordered: the central $\mathrm{Si}$ atom and the $\mathrm{Cl}$ atoms

Table 2

Experimental details.

\begin{tabular}{ll}
\hline Crystal data & \\
Chemical formula & $\mathrm{Cl}_{12} \mathrm{Si}_{5} \cdot \mathrm{C}_{6} \mathrm{H}_{6}$ \\
$M_{\mathrm{r}}$ & 643.96 \\
Crystal system, space group & Tetragonal, $P 4_{1} 22$ \\
Temperature $(\mathrm{K})$ & 173 \\
$a, c(\AA)$ & $11.9633(4), 33.7848(16)$ \\
$V\left(\AA^{3}\right)$ & $4835.3(4)$ \\
$Z$ & 8 \\
Radiation type & $\mathrm{Mo} \mathrm{K \alpha}$ \\
$\mu\left(\mathrm{mm}^{-1}\right)$ & 1.62 \\
Crystal size $(\mathrm{mm})$ & $0.28 \times 0.18 \times 0.16$
\end{tabular}

Data collection

Diffractometer

Absorption correction

$T_{\min }, T_{\max }$

No. of measured, independent and observed $[I>2 \sigma(I)]$ reflections

$R_{\text {int }}$

$(\sin \theta / \lambda)_{\max }\left(\AA^{-1}\right)$

Stoe IPDS II two-circle

Multi-scan ( $X$-AREA; Stoe \& Cie, 2001)

$0.803,1.0$

$39180,5189,4790$

0.047

0.642

Refinement

$R\left[F^{2}>2 \sigma\left(F^{2}\right)\right], w R\left(F^{2}\right), S$

No. of reflections

No. of parameters

No. of restraints

$\mathrm{H}$-atom treatment

$\Delta \rho_{\max }, \Delta \rho_{\min }\left(\mathrm{e} \AA^{-3}\right)$

Absolute structure

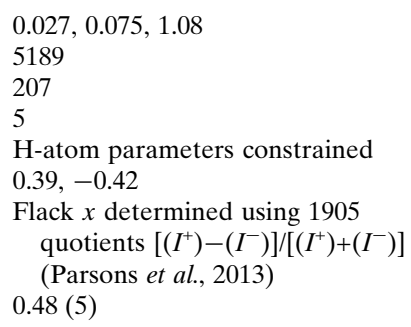
$0.48(5)$

Absolute structure parameter

Computer programs: $X$-AREA (Stoe \& Cie, 2001), SHELXS97 (Sheldrick, 2008), XP in SHELXTL-Plus (Sheldrick, 2008), SHELXL2018 (Sheldrick, 2015) and publCIF (Westrip, 2010). 
themselves are not disordered. In (III), the $\mathrm{Si}_{5} \mathrm{Cl}_{12}$ molecule is located on a special position of site symmetry 23 . The central $\mathrm{Si}$ atom is located at the intersection of the twofold and the threefold rotation axes (the twofold rotation axis coincides with a $\overline{4}$ axis). The $\mathrm{Si}-\mathrm{Si}$ and $\mathrm{Si}-\mathrm{Cl}$ bond lengths in all three structures agree well (Table 1).

\section{Synthesis and crystallization}

The perchlorinated neopentasilane (I) was synthesized according to a literature procedure (Kaczmarczyk \& Urry, 1960). Single crystals of $\mathrm{Si}\left(\mathrm{SiCl}_{3}\right)_{4} \cdot \mathrm{C}_{6} \mathrm{H}_{6}$ were grown from a solution of $\mathrm{Si}\left(\mathrm{SiCl}_{3}\right)_{4}$ in benzene after one week at room temperature.

$\mathbf{S i}\left(\mathbf{S i C l}_{3}\right)_{4}(\mathbf{I}) .{ }^{29} \mathrm{Si}\left\{{ }^{1} \mathrm{H}\right\} \mathrm{NMR}\left(\mathrm{C}_{6} \mathrm{D}_{6}\right.$, external TMS $): \delta=$ $-80.9\left[\mathrm{Si}\left(\mathrm{SiCl}_{3}\right)_{4}\right], \delta=3.5\left[\mathrm{Si}\left(\mathrm{SiCl}_{3}\right)_{4}\right]$.

\section{Refinement}

Crystal data, data collection and structure refinement details are summarized in Table 1 . The $\mathrm{H}$ atoms were refined using a riding model with $\mathrm{C}-\mathrm{H}=0.95 \AA$ and with $U_{\text {iso }}(\mathrm{H})=$ $1.2 U_{\text {eq }}(\mathrm{C})$. One of the benzene molecules is disordered over two equally occupied orientations: its carbon atoms were isotropically refined. The $\mathrm{C}-\mathrm{C}$ distances in the non-disordered benzene molecule were restrained to 1.390 (2) $\AA$. The crystal chosen for data collection was found to crystallize as a racemic twin.

\section{References}

Cannady, J. P. \& Zhou, X. (2008). WO2008051328.

Fleming, D. K. (1972). Acta Cryst. B28, 1233-1236.

Kaczmarczyk, A. \& Urry, G. (1960). J. Am. Chem. Soc. 82, 751-752.

Meyer-Wegner, F., Nadj, A., Bolte, M., Auner, N., Wagner, M., Holthausen, M. C. \& Lerner, H.-W. (2011). Chem. Eur. J. 17, 47154719.

Nishimura, Y., Eguchi, T., Matsuda, H., Haruta, M., Hirai, Y. \& Nakagiri, T. (1985). JP, 60219733 A 19851102.

Parsons, S., Flack, H. D. \& Wagner, T. (2013). Acta Cryst. B69, 249259.

Sheldrick, G. M. (2008). Acta Cryst. A64, 112-122.

Sheldrick, G. M. (2015). Acta Cryst. C71, 3-8.

Stoe \& Cie (2001). X-AREA. Stoe \& Cie, Darmstadt, Germany.

Tillmann, J., Meyer-Wegner, F., Nadj, A., Becker-Baldus, J., Sinke, T., Bolte, M., Holthausen, M. C., Wagner, M. \& Lerner, H.-W. (2012). Inorg. Chem. 51, 8599-8606.

Urry, G. (1970). Acc. Chem. Res. 3, 306-312.

Westrip, S. P. (2010). J. Appl. Cryst. 43, 920-925. 


\section{supporting information}

Acta Cryst. (2020). E76, 261-263 [https://doi.org/10.1107/S2056989020000900]

\section{A new pseudopolymorph of perchlorinated neopentasilane: the benzene monosolvate $\mathrm{Si}\left(\mathrm{SiCl}_{3}\right)_{4} \cdot \mathrm{C}_{6} \mathrm{H}_{6}$}

\section{Jan Tillmann, Hans-Wolfram Lerner and Michael Bolte}

Computing details

Data collection: $X$-AREA (Stoe \& Cie, 2001); cell refinement: $X$-AREA (Stoe \& Cie, 2001); data reduction: $X$-AREA (Stoe \& Cie, 2001); program(s) used to solve structure: SHELXS97 (Sheldrick, 2008); program(s) used to refine structure:

SHELXL2018 (Sheldrick, 2015); molecular graphics: XP in SHELXTL-Plus (Sheldrick, 2008); software used to prepare material for publication: SHELXL2018 (Sheldrick, 2015) and publCIF (Westrip, 2010).

Dodecachloropentasilane benzene monosolvate

\section{Crystal data}

$\mathrm{Cl}_{12} \mathrm{Si}_{5} \cdot \mathrm{C}_{6} \mathrm{H}_{6}$

$M_{r}=643.96$

Tetragonal, $P 4_{1} 22$

$a=11.9633(4) \AA$

$c=33.7848(16) \AA$

$V=4835.3(4) \AA^{3}$

$Z=8$

$F(000)=2528$

\section{Data collection}

Stoe IPDS II two-circle diffractometer

$\omega$ scans

Absorption correction: multi-scan

(X-AREA; Stoe \& Cie, 2001)

$T_{\min }=0.803, T_{\max }=1.0$

39180 measured reflections

\section{Refinement}

Refinement on $F^{2}$

Least-squares matrix: full

$R\left[F^{2}>2 \sigma\left(F^{2}\right)\right]=0.027$

$w R\left(F^{2}\right)=0.075$

$S=1.08$

5189 reflections

207 parameters

5 restraints

Primary atom site location: structure-invariant direct methods

Hydrogen site location: mixed
$D_{\mathrm{x}}=1.769 \mathrm{Mg} \mathrm{m}^{-3}$

Mo $K \alpha$ radiation, $\lambda=0.71073 \AA$

Cell parameters from 62511 reflections

$\theta=1.9-27.6^{\circ}$

$\mu=1.62 \mathrm{~mm}^{-1}$

$T=173 \mathrm{~K}$

Block, colourless

$0.28 \times 0.18 \times 0.16 \mathrm{~mm}$

5189 independent reflections

4790 reflections with $I>2 \sigma(I)$

$R_{\text {int }}=0.047$

$\theta_{\max }=27.1^{\circ}, \theta_{\min }=1.8^{\circ}$

$h=-14 \rightarrow 15$

$k=-15 \rightarrow 15$

$l=-43 \rightarrow 43$

$\mathrm{H}$-atom parameters constrained

$w=1 /\left[\sigma^{2}\left(F_{\mathrm{o}}^{2}\right)+(0.0375 P)^{2}+2.8317 P\right]$

where $P=\left(F_{\mathrm{o}}{ }^{2}+2 F_{\mathrm{c}}{ }^{2}\right) / 3$

$(\Delta / \sigma)_{\max }=0.001$

$\Delta \rho_{\max }=0.39 \mathrm{e} \AA^{-3}$

$\Delta \rho_{\min }=-0.42$ e $\AA^{-3}$

Absolute structure: Flack $x$ determined using 1905 quotients $\left[\left(I^{+}\right)-(I)\right] /\left[\left(I^{+}\right)+\left(I^{-}\right)\right]$(Parsons et al., 2013)

Absolute structure parameter: 0.48 (5) 


\section{Special details}

Geometry. All esds (except the esd in the dihedral angle between two 1.s. planes) are estimated using the full covariance matrix. The cell esds are taken into account individually in the estimation of esds in distances, angles and torsion angles; correlations between esds in cell parameters are only used when they are defined by crystal symmetry. An approximate (isotropic) treatment of cell esds is used for estimating esds involving l.s. planes.

Fractional atomic coordinates and isotropic or equivalent isotropic displacement parameters $\left(\AA^{2}\right)$

\begin{tabular}{|c|c|c|c|c|c|}
\hline & $x$ & $y$ & $z$ & $U_{\text {iso }} * / U_{\text {eq }}$ & Occ. $(<1)$ \\
\hline Sil & $0.54973(7)$ & $0.45027(7)$ & 0.125000 & $0.0222(3)$ & \\
\hline $\mathrm{Si} 2$ & $0.38834(7)$ & $0.45056(7)$ & $0.08678(2)$ & 0.02447 (19) & \\
\hline $\mathrm{Si} 3$ & $0.54972(8)$ & $0.29277(8)$ & $0.16530(3)$ & $0.0264(2)$ & \\
\hline $\mathrm{Cl} 21$ & $0.40377(8)$ & $0.34525(8)$ & $0.04060(2)$ & $0.0400(2)$ & \\
\hline $\mathrm{Cl} 22$ & $0.25695(7)$ & $0.39988(8)$ & $0.11990(3)$ & $0.0387(2)$ & \\
\hline $\mathrm{Cl} 23$ & $0.35776(8)$ & $0.60671(7)$ & $0.06658(3)$ & $0.0384(2)$ & \\
\hline $\mathrm{Cl} 31$ & $0.49743(8)$ & $0.15929(7)$ & $0.13366(3)$ & $0.0408(2)$ & \\
\hline $\mathrm{Cl} 32$ & $0.70602(8)$ & $0.26452(9)$ & $0.18579(3)$ & $0.0453(2)$ & \\
\hline $\mathrm{Cl} 33$ & $0.44514(8)$ & $0.31660(9)$ & $0.21128(3)$ & $0.0412(2)$ & \\
\hline $\mathrm{Si} 4$ & $0.04988(7)$ & $-0.04988(7)$ & 0.125000 & $0.0225(3)$ & \\
\hline $\mathrm{Si} 5$ & $0.05165(7)$ & $0.11311(7)$ & $0.16232(2)$ & $0.02420(19)$ & \\
\hline Si6 & $0.20502(8)$ & $-0.05209(8)$ & $0.08356(3)$ & $0.0263(2)$ & \\
\hline $\mathrm{Cl} 51$ & $-0.05058(7)$ & $0.09833(8)$ & $0.20938(2)$ & $0.0382(2)$ & \\
\hline $\mathrm{Cl} 52$ & $-0.00287(8)$ & $0.24225(7)$ & $0.12875(3)$ & $0.0367(2)$ & \\
\hline $\mathrm{Cl} 53$ & $0.20867(7)$ & $0.14553(8)$ & $0.18124(3)$ & $0.0379(2)$ & \\
\hline $\mathrm{Cl} 61$ & $0.23134(9)$ & $0.10282(8)$ & $0.06166(3)$ & $0.0449(2)$ & \\
\hline $\mathrm{Cl} 62$ & $0.17856(9)$ & $-0.15973(8)$ & $0.03862(2)$ & $0.0414(2)$ & \\
\hline $\mathrm{Cl63}$ & $0.34091(8)$ & -0.10198 (9) & $0.11430(3)$ & $0.0424(2)$ & \\
\hline $\mathrm{C} 1$ & 0.000000 & $0.3434(6)$ & 0.000000 & $0.100(4)$ & \\
\hline H1 & 0.000000 & 0.263981 & 0.000000 & $0.120^{*}$ & \\
\hline $\mathrm{C} 2$ & $0.0511(5)$ & $0.4024(4)$ & $0.03030(13)$ & $0.094(2)$ & \\
\hline $\mathrm{H} 2$ & 0.087169 & 0.363505 & 0.051231 & $0.113^{*}$ & \\
\hline $\mathrm{C} 3$ & $0.0495(5)$ & $0.5176(4)$ & $0.03001(14)$ & $0.090(2)$ & \\
\hline H3 & 0.083717 & 0.556596 & 0.051284 & $0.107^{*}$ & \\
\hline $\mathrm{C} 4$ & 0.000000 & $0.5786(7)$ & 0.000000 & $0.087(3)$ & \\
\hline H4 & 0.000000 & 0.657984 & 0.000000 & $0.104^{*}$ & \\
\hline $\mathrm{C} 5$ & $0.5409(8)$ & $0.8628(10)$ & $0.0236(3)$ & $0.051(2)^{*}$ & 0.5 \\
\hline H5 & 0.570943 & 0.806236 & 0.040196 & $0.062^{*}$ & 0.5 \\
\hline C6 & $0.5561(8)$ & $0.9738(11)$ & $0.0335(3)$ & $0.050(2)^{*}$ & 0.5 \\
\hline H6 & 0.594385 & 0.992910 & 0.057205 & $0.059^{*}$ & 0.5 \\
\hline $\mathrm{C} 7$ & $0.5158(9)$ & $1.0566(8)$ & $0.0092(3)$ & $0.054(3)^{*}$ & 0.5 \\
\hline H7 & 0.527035 & 1.132987 & 0.015732 & $0.065^{*}$ & 0.5 \\
\hline $\mathrm{C}^{\prime}$ & $0.5145(10)$ & $0.8339(8)$ & $0.0086(3)$ & $0.057(3)^{*}$ & 0.5 \\
\hline $\mathrm{H} 5^{\prime}$ & 0.526088 & 0.757264 & 0.014687 & $0.068^{*}$ & 0.5 \\
\hline C6 ${ }^{\prime}$ & $0.5559(9)$ & $0.9177(12)$ & $0.0335(3)$ & $0.058(2)^{*}$ & 0.5 \\
\hline $\mathrm{H} 6^{\prime}$ & 0.594991 & 0.897299 & 0.056942 & $0.069^{*}$ & 0.5 \\
\hline $\mathrm{C} 7^{\prime}$ & $0.5410(10)$ & $1.0290(11)$ & 0.0247 (4) & $0.062(3)^{*}$ & 0.5 \\
\hline $\mathrm{H} 7^{\prime}$ & 0.569534 & 1.085379 & 0.041783 & $0.074 *$ & 0.5 \\
\hline
\end{tabular}


Atomic displacement parameters $\left(\AA^{2}\right)$

\begin{tabular}{|c|c|c|c|c|c|c|}
\hline & $U^{11}$ & $U^{22}$ & $U^{33}$ & $U^{12}$ & $U^{13}$ & $U^{23}$ \\
\hline Sil & $0.0213(4)$ & 0.0213 & $0.0241(6)$ & $0.0004(5)$ & $0.0008(3)$ & $0.0008(3)$ \\
\hline $\mathrm{Si} 2$ & $0.0245(4)$ & $0.0238(4)$ & $0.0251(4)$ & $0.0000(3)$ & -0.0010 & 0.0009 (3) \\
\hline $\mathrm{Si} 3$ & $0.0252(4)$ & $0.0247(4)$ & $0.0294(4)$ & 0.0001 (4) & $-0.0007(3)$ & $0.0046(3)$ \\
\hline $\mathrm{Cl} 21$ & $0.0521(5)$ & $0.0364(5)$ & $0.0316(4)$ & -0.0014 & $0.0003(4)$ & $-0.0084(3)$ \\
\hline $\mathrm{Cl} 22$ & $0.0269(4)$ & $0.0476(5)$ & $0.0418(4)$ & $-0.0054(4)$ & $0.0051(3)$ & $0.0042(4)$ \\
\hline $\mathrm{Cl} 23$ & $0.0464(5)$ & 0.0278 & $0.0411(4)$ & 0.0058 & $-0.0079(4)$ & $0.0060(3)$ \\
\hline $\mathrm{Cl} 31$ & $0.0452(5)$ & 0.0268 & $0.0503(5)$ & $-0.0048(4)$ & -0.0003 & $-0.0032(4)$ \\
\hline $\mathrm{Cl} 32$ & $0.0317(4)$ & $0.0461(5)$ & $0.0579(5)$ & 0.0068 & $-0.0113(4)$ & $0.0116(4)$ \\
\hline $\mathrm{Cl} 33$ & $0.0406(5)$ & $0.0529(6)$ & $0.0302(4)$ & -0.0059 & 0.0065 (4) & $0.0031(4)$ \\
\hline $\mathrm{Si} 4$ & $0.0214(4)$ & 0.0214 & $0.0245(6)$ & $0.0000(5)$ & $0.0006(3)$ & $0.0006(3)$ \\
\hline $\mathrm{Si} 5$ & $0.0236(4)$ & 0.0242 & $0.0248(4)$ & $-0.0008(3)$ & -0.0010 & $-0.0011(3)$ \\
\hline $\mathrm{Si} 6$ & $0.0248(4)$ & 0.0259 (4) & $0.0282(4)$ & 0.0001 & 0.0038 & $0.0010(3)$ \\
\hline $\mathrm{Cl} 51$ & $0.0366(5)$ & $0.0486(5)$ & $0.0295(4)$ & 0.0010 & $0.0072(3)$ & $-0.0013(4)$ \\
\hline $\mathrm{Cl} 52$ & $0.0436(5)$ & 0.0268 & $0.0398(4)$ & 0.0039 & -0.0050 & $0.0042(3)$ \\
\hline $\mathrm{Cl} 53$ & $0.0282(4)$ & $0.0428(5)$ & $0.0429(4)$ & $-0.0063(4)$ & -0.0069 & $-0.0033(4)$ \\
\hline $\mathrm{Cl} 61$ & $0.0489(5)$ & 0.0325 & $0.0533(5)$ & $-0.0063(4)$ & $0.0126(4)$ & $0.0108(4)$ \\
\hline Cl62 & $0.0520(5)$ & $0.0404(5)$ & $0.0317(4)$ & 0.0053 & $0.0022(4)$ & $-0.0084(4)$ \\
\hline $\mathrm{Cl} 63$ & $0.0274(4)$ & 0.0505 & $0.0494(5)$ & 0.0067 (4) & -0.0039 & $0.0003(4)$ \\
\hline $\mathrm{C} 1$ & $0.074(6)$ & $0.043(4)$ & $0.184(11)$ & 0.000 & $0.056(7)$ & 0.000 \\
\hline $\mathrm{C} 2$ & 0.068 & $0.147(7)$ & $0.067(4)$ & $0.011(4)$ & $0.001(3)$ & $0.055(4)$ \\
\hline $\mathrm{C} 3$ & 0.070 & $0.138(6)$ & $0.060(3)$ & $-0.026(4)$ & $0.006(3)$ & $-0.037(4)$ \\
\hline $\mathrm{C} 4$ & $0.067(5)$ & $0.068(5)$ & $0.125(8)$ & 0.000 & $0.040(5)$ & 0.000 \\
\hline
\end{tabular}

Geometric parameters $\left(\AA,{ }^{\circ}\right)$

\begin{tabular}{|c|c|c|c|}
\hline $\mathrm{Si} 1-\mathrm{Si} 2$ & $2.3227(11)$ & $\mathrm{Si} 6-\mathrm{Cl} 161$ & $2.0202(13)$ \\
\hline $\mathrm{Si} 1-\mathrm{Si} 2^{\mathrm{i}}$ & $2.3228(11)$ & $\mathrm{C} 1-\mathrm{C} 2$ & $1.3855(18)$ \\
\hline $\mathrm{Si} 1-\mathrm{Si}^{\mathrm{i}}$ & $2.3246(11)$ & $\mathrm{C} 1-\mathrm{C} 2^{\mathrm{iii}}$ & $1.3855(18)$ \\
\hline $\mathrm{Si} 1-\mathrm{Si} 3$ & $2.3247(11)$ & $\mathrm{C} 1-\mathrm{H} 1$ & 0.9500 \\
\hline $\mathrm{Si} 2-\mathrm{Cl} 21$ & $2.0138(12)$ & $\mathrm{C} 2-\mathrm{C} 3$ & $1.378(3)$ \\
\hline $\mathrm{Si} 2-\mathrm{Cl} 23$ & $2.0221(12)$ & $\mathrm{C} 2-\mathrm{H} 2$ & 0.9500 \\
\hline $\mathrm{Si} 2-\mathrm{Cl} 22$ & $2.0225(12)$ & $\mathrm{C} 3-\mathrm{C} 4$ & $1.3824(18)$ \\
\hline $\mathrm{Si} 3-\mathrm{Cl} 33$ & $2.0150(13)$ & $\mathrm{C} 3-\mathrm{H} 3$ & 0.9500 \\
\hline $\mathrm{Si} 3-\mathrm{Cl} 31$ & $2.0209(13)$ & $\mathrm{C} 4-\mathrm{H} 4$ & 0.9500 \\
\hline $\mathrm{Si} 3-\mathrm{Cl} 32$ & $2.0223(13)$ & $\mathrm{C} 5-\mathrm{C} 6$ & $1.382(15)$ \\
\hline $\mathrm{Si} 4-\mathrm{Si} 5$ & $2.3221(11)$ & $\mathrm{C} 5-\mathrm{H} 5$ & 0.9500 \\
\hline $\mathrm{Si} 4-\mathrm{Si}^{\mathrm{ii}}$ & $2.3222(11)$ & $\mathrm{C} 6-\mathrm{C} 7$ & $1.375(14)$ \\
\hline $\mathrm{Si} 4-\mathrm{Si} 6$ & $2.3250(11)$ & $\mathrm{C} 6-\mathrm{H} 6$ & 0.9500 \\
\hline $\mathrm{Si} 4-\mathrm{Si}^{\mathrm{ii}}$ & $2.3251(11)$ & $\mathrm{C} 7-\mathrm{H} 7$ & 0.9500 \\
\hline $\mathrm{Si} 5-\mathrm{Cl} 51$ & $2.0138(12)$ & $\mathrm{C} 5^{\prime}-\mathrm{C} 6^{\prime}$ & $1.399(15)$ \\
\hline $\mathrm{Si} 5-\mathrm{Cl} 53$ & $2.0218(12)$ & $\mathrm{C} 5^{\prime}-\mathrm{H} 5^{\prime}$ & 0.9501 \\
\hline $\mathrm{Si} 5-\mathrm{Cl} 52$ & $2.0243(12)$ & $\mathrm{C}^{\prime}-\mathrm{C} 7^{\prime}$ & $1.376(15)$ \\
\hline $\mathrm{Si} 6-\mathrm{Cl} 62$ & $2.0158(13)$ & $\mathrm{C}^{\prime}-\mathrm{H}^{\prime}$ & 0.9500 \\
\hline $\mathrm{Si} 6-\mathrm{Cl} 63$ & $2.0194(13)$ & $\mathrm{C} 7^{\prime}-\mathrm{H} 7^{\prime}$ & 0.9500 \\
\hline
\end{tabular}




\begin{tabular}{|c|c|c|c|}
\hline $\mathrm{Si} 2-\mathrm{Si} 1-\mathrm{Si} 2^{\mathrm{i}}$ & $107.85(6)$ & $\mathrm{C} 2-\mathrm{C} 1-\mathrm{C} 2^{\mathrm{iii}}$ & $118.8(7)$ \\
\hline $\mathrm{Si} 2-\mathrm{Si} 1-\mathrm{Si} 3^{\mathrm{i}}$ & $110.38(3)$ & $\mathrm{C} 2-\mathrm{C} 1-\mathrm{H} 1$ & 120.6 \\
\hline $\mathrm{Si} 2^{\mathrm{i}}-\mathrm{Si} 1-\mathrm{Si}^{\mathrm{i}}$ & $109.07(3)$ & $\mathrm{C} 22^{\mathrm{iii}}-\mathrm{C} 1-\mathrm{H} 1$ & 120.6 \\
\hline $\mathrm{Si} 2-\mathrm{Si} 1-\mathrm{Si} 3$ & $109.07(3)$ & $\mathrm{C} 3-\mathrm{C} 2-\mathrm{C} 1$ & $119.9(6)$ \\
\hline $\mathrm{Si} 2 \mathrm{i}-\mathrm{Si} 1-\mathrm{Si} 3$ & $110.37(3)$ & $\mathrm{C} 3-\mathrm{C} 2-\mathrm{H} 2$ & 120.1 \\
\hline $\mathrm{Si} 3^{\mathrm{i}}-\mathrm{Si} 1-\mathrm{Si} 3$ & $110.06(7)$ & $\mathrm{C} 1-\mathrm{C} 2-\mathrm{H} 2$ & 120.1 \\
\hline $\mathrm{Cl} 21-\mathrm{Si} 2-\mathrm{Cl} 23$ & $109.46(5)$ & $\mathrm{C} 2-\mathrm{C} 3-\mathrm{C} 4$ & $122.6(6)$ \\
\hline $\mathrm{Cl} 21-\mathrm{Si} 2-\mathrm{Cl} 22$ & $108.20(6)$ & $\mathrm{C} 2-\mathrm{C} 3-\mathrm{H} 3$ & 118.7 \\
\hline $\mathrm{Cl} 23-\mathrm{Si} 2-\mathrm{Cl} 22$ & $108.85(6)$ & $\mathrm{C} 4-\mathrm{C} 3-\mathrm{H} 3$ & 118.7 \\
\hline $\mathrm{C} 121-\mathrm{Si} 2-\mathrm{Si} 1$ & $110.71(5)$ & $\mathrm{C} 3^{\mathrm{iii}-\mathrm{C} 4-\mathrm{C} 3}$ & $116.3(7)$ \\
\hline $\mathrm{Cl} 23-\mathrm{Si} 2-\mathrm{Si} 1$ & $109.84(5)$ & $\mathrm{C} 3^{\mathrm{iii}-\mathrm{C} 4-\mathrm{H} 4}$ & 121.9 \\
\hline $\mathrm{Cl} 22-\mathrm{Si} 2-\mathrm{Si} 1$ & $109.75(4)$ & $\mathrm{C} 3-\mathrm{C} 4-\mathrm{H} 4$ & 121.9 \\
\hline $\mathrm{Cl} 33-\mathrm{Si} 3-\mathrm{Cl} 31$ & $109.11(6)$ & $\mathrm{C} 6-\mathrm{C} 5-\mathrm{C} 5^{\mathrm{iv}}$ & $106.0(6)$ \\
\hline $\mathrm{Cl} 33-\mathrm{Si} 3-\mathrm{Cl} 32$ & $109.50(6)$ & $\mathrm{C} 6-\mathrm{C} 5-\mathrm{H} 5$ & 119.4 \\
\hline $\mathrm{Cl} 31-\mathrm{Si} 3-\mathrm{Cl} 32$ & $109.58(6)$ & $\mathrm{C}^{\mathrm{iv}}-\mathrm{C} 5-\mathrm{H} 5$ & 134.6 \\
\hline $\mathrm{Cl} 33-\mathrm{Si} 3-\mathrm{Si} 1$ & $109.69(5)$ & $\mathrm{C} 5-\mathrm{C} 6-\mathrm{C} 7$ & $120.0(9)$ \\
\hline $\mathrm{Cl} 31-\mathrm{Si} 3-\mathrm{Si} 1$ & $109.31(5)$ & $\mathrm{C} 5-\mathrm{C} 6-\mathrm{C}^{\mathrm{iv}}$ & $104.5(8)$ \\
\hline $\mathrm{Cl} 32-\mathrm{Si} 3-\mathrm{Si} 1$ & $109.63(5)$ & $\mathrm{C} 5-\mathrm{C} 6-\mathrm{H} 6$ & 120.0 \\
\hline $\mathrm{Si} 5-\mathrm{Si} 4-\mathrm{Si} 5^{\mathrm{ii}}$ & $108.07(6)$ & $\mathrm{C} 7-\mathrm{C} 6-\mathrm{H} 6$ & 120.0 \\
\hline $\mathrm{Si} 5-\mathrm{Si} 4-\mathrm{Si} 6$ & $109.22(3)$ & $\mathrm{C}^{\mathrm{iv}}-\mathrm{C} 6-\mathrm{H} 6$ & 135.5 \\
\hline $\mathrm{Si} 55^{\mathrm{ii}}-\mathrm{Si} 4-\mathrm{Si} 6$ & $110.07(3)$ & $\mathrm{C} 7^{\mathrm{iv}}-\mathrm{C} 7-\mathrm{C} 6$ & $133.9(6)$ \\
\hline $\mathrm{Si} 5-\mathrm{Si} 4-\mathrm{Si}^{\mathrm{ii}}$ & $110.08(3)$ & $\mathrm{C} 6-\mathrm{C} 7-\mathrm{C}^{\mathrm{iv}}$ & $103.4(9)$ \\
\hline $\mathrm{Si} 5^{\mathrm{ii}}-\mathrm{Si} 4-\mathrm{Si} 6^{\mathrm{ii}}$ & $109.22(3)$ & $\mathrm{C}^{\mathrm{iv}}-\mathrm{C} 7-\mathrm{H} 7$ & 105.9 \\
\hline $\mathrm{Si} 6-\mathrm{Si} 4-\mathrm{Si}^{\mathrm{ii}}$ & $110.15(7)$ & $\mathrm{C} 6-\mathrm{C} 7-\mathrm{H} 7$ & 120.2 \\
\hline $\mathrm{Cl} 151-\mathrm{Si} 5-\mathrm{Cl} 53$ & $109.35(5)$ & $\mathrm{C} 6^{\mathrm{iv}}-\mathrm{C} 7-\mathrm{H} 7$ & 136.4 \\
\hline $\mathrm{Cl} 151-\mathrm{Si} 5-\mathrm{Cl} 52$ & $108.28(6)$ & $\mathrm{C}^{\prime \text { iv }}-\mathrm{C} 5^{\prime}-\mathrm{C} 6^{\prime}$ & $134.3(6)$ \\
\hline $\mathrm{Cl} 153-\mathrm{Si} 5-\mathrm{Cl} 52$ & $109.28(6)$ & $\mathrm{C}^{\prime \text { iv }}-\mathrm{C} 5^{\prime}-\mathrm{H} 5^{\prime}$ & 105.1 \\
\hline $\mathrm{Cl} 151-\mathrm{Si} 5-\mathrm{Si} 4$ & $110.45(5)$ & $\mathrm{C} 6^{\prime}-\mathrm{C} 5^{\prime}-\mathrm{H} 5^{\prime}$ & 120.6 \\
\hline $\mathrm{Cl} 153-\mathrm{Si} 5-\mathrm{Si} 4$ & $109.95(5)$ & $\mathrm{C}^{\prime \text { iv }}-\mathrm{C} 5^{\prime}-\mathrm{H} 5^{\prime}$ & 136.3 \\
\hline $\mathrm{Cl} 152-\mathrm{Si} 5-\mathrm{Si} 4$ & $109.50(5)$ & $\mathrm{C} 7^{\prime}-\mathrm{C} 6^{\prime}-\mathrm{C} 5^{\prime}$ & $121.1(10)$ \\
\hline $\mathrm{Cl} 162-\mathrm{Si} 6-\mathrm{C} 163$ & $108.97(6)$ & $\mathrm{C} 7^{\prime}-\mathrm{C} 6^{\prime}-\mathrm{H} 6^{\prime}$ & 119.4 \\
\hline $\mathrm{Cl} 162-\mathrm{Si} 6-\mathrm{Cl} 61$ & $109.56(6)$ & $\mathrm{C} 5^{\prime}-\mathrm{C} 6^{\prime}-\mathrm{H} 6^{\prime}$ & 119.4 \\
\hline $\mathrm{Cl} 63-\mathrm{Si} 6-\mathrm{Cl} 61$ & $109.51(6)$ & $\mathrm{C} 5^{\prime \text { iv }}-\mathrm{C} 6^{\prime}-\mathrm{H} 6^{\prime}$ & 134.0 \\
\hline $\mathrm{Cl} 62-\mathrm{Si} 6-\mathrm{Si} 4$ & $109.60(5)$ & $\mathrm{C} 6^{\prime}-\mathrm{C} 7^{\prime}-\mathrm{C} 7^{\text {iv }}$ & $104.6(7)$ \\
\hline $\mathrm{Cl} 63-\mathrm{Si} 6-\mathrm{Si} 4$ & $109.65(5)$ & $\mathrm{C} 6^{\prime}-\mathrm{C} 7^{\prime}-\mathrm{H} 7^{\prime}$ & 120.6 \\
\hline $\mathrm{Cl} 61-\mathrm{Si} 6-\mathrm{Si} 4$ & $109.54(5)$ & $\mathrm{C}^{\prime \text { iv }}-\mathrm{C} 7^{\prime}-\mathrm{H} 7^{\prime}$ & 134.8 \\
\hline $\mathrm{C} 2{ }^{\mathrm{iii}-} \mathrm{C} 1-\mathrm{C} 2-\mathrm{C} 3$ & $0.6(4)$ & $\mathrm{C}^{\mathrm{iv}}-\mathrm{C} 6-\mathrm{C} 7-\mathrm{C}^{\mathrm{iv}}$ & $-1(2)$ \\
\hline $\mathrm{C} 1-\mathrm{C} 2-\mathrm{C} 3-\mathrm{C} 4$ & $-1.2(9)$ & $\mathrm{C} 5^{\text {,iv }-}-\mathrm{C} 5^{\prime}-\mathrm{C} 6^{\prime}-\mathrm{C} 7^{\prime}$ & $0(4)$ \\
\hline $\mathrm{C} 2-\mathrm{C} 3-\mathrm{C} 4-\mathrm{C} 3^{\mathrm{iii}}$ & $0.6(4)$ & $\mathrm{C} 6^{\prime \text { iv }}-\mathrm{C} 5^{\prime}-\mathrm{C} 6^{\prime}-\mathrm{C} 7^{\prime}$ & $0.0(10)$ \\
\hline $\mathrm{C} 5^{\mathrm{iv}}-\mathrm{C} 5-\mathrm{C} 6-\mathrm{C} 7$ & $-1.7(13)$ & $\mathrm{C} 6^{\text {iv }}-\mathrm{C}^{\prime}-\mathrm{C} 6^{\prime}-\mathrm{C} 5^{\text {iv }}$ & $0(3)$ \\
\hline $\mathrm{C}^{\mathrm{iv}}-\mathrm{C} 5-\mathrm{C} 6-\mathrm{C}^{\mathrm{iv}}$ & $-1.2(10)$ & $\mathrm{C} 5^{\prime}-\mathrm{C} 6^{\prime}-\mathrm{C} 7^{\prime}-\mathrm{C} 7^{\text {iv }}$ & $-0.1(14)$ \\
\hline $\mathrm{C} 5-\mathrm{C} 6-\mathrm{C} 7-\mathrm{C}^{\mathrm{iv}}$ & $2(3)$ & $\mathrm{C}^{\prime \text {,iv }}-\mathrm{C} 6^{\prime}-\mathrm{C}^{\prime}-\mathrm{C}^{\prime \text { iv }}$ & $-0.2(11)$ \\
\hline $\mathrm{C} 5-\mathrm{C} 6-\mathrm{C} 7-\mathrm{C}^{\mathrm{iv}}$ & $1.0(9)$ & & \\
\hline
\end{tabular}

Symmetry codes: (i) $-y+1,-x+1,-z+1 / 4$; (ii) $-y,-x,-z+1 / 4$; (iii) $-x, y,-z$; (iv) $-x+1, y,-z$. 